\title{
Aspectos quirúrgicos en los terceros y cuartos retrasplantes renales
}

\author{
J.L.Gutiérrez Baños, E. Rodrigo Calabia*, M.H. Rebollo Rodrigo**, J.A. Portillo Martín, \\ A. Roca Edreira, M.Á. Correas Gómez, J. Ignacio del Valle Schaan, C. Aguilera Tubet, \\ F. Ruiz Izquierdo, R. Ballestero Diego, B. Martín García
}

Servicios de Urología, *Nefrología y **Medicina Preventiva. Hospital Universitario Marqués de Valdecilla. Santander.

Actas Urol Esp 2005; 29 (2): 212-216

\section{RESUMEN}

ASPECTOS QUIRURGICOS EN LOS TERCEROS Y CUARTOS RETRASPLANTES RENALES

Introducción y objetivos: El fracaso de un injerto renal previo constituye la cuarta parte de los pacientes en lista de espera para trasplante renal. Si bien los resultados de los primeros y segundos trasplantes hacen que sean el tratamiento prioritario de la insuficiencia renal crónica, no es tan evidente que el tercer o más trasplante sea la mejor elección especialmente dada la escasez de órganos. Revisamos nuestra experiencia en pacientes con tres o más trasplantes renales con especial atención a los aspectos quirúrgicos y evolución del injerto.

Material y método: Entre 1975 y diciembre de 2003 hemos realizado 1.364 trasplantes renales. Hacemos una revisión retrospectiva de los 34 pacientes con 3 trasplantes y 5 con 4 trasplantes. Analizamos fundamentalmente la técnica y complicaciones quirúrgicas y la evolución del injerto.

Resultados: La edad media fue de 42 años (21-65). La media de incompatibilidades entre donante y receptor fue de 3,2. Todos, excepto 1 caso de donante vivo, fueron donantes cadáveres, la mayoría multiorgánicos. El tiempo medio desde el trasplante previo fue de 5 años ( 3 días-17 años) y entre la última cirugía en la fosa ilíaca reutilizada y el trasplante fue de 9 años ( 3 días y 17,5 años). En todos los casos se utilizó una incisión lumboilíaca iterativa ( 25 derecha, 11 izquierda, 3 no consta); el mayor problema quirúrgico fue la existencia de fibrosis en la fosa ilíaca. La duración media de la intervención fue de 166 minutos (100-300). En 13 pacientes (33\%) se realizó trasplantectomía en el mismo acto quirúrgico. La anastomosis vascular se hizo en los vasos ilíacos comunes (50\%) o externos (50\%). La ureteroneocistostomía se hizo mediante técnica extravesical excepto en 1 caso con derivación a piel. Complicaciones vasculares: 4 hemorragias (1 exitus), 3 trombosis venosas y 2 arteriales. Hubo 1 absceso del lecho secundario a una fístula intestinal. Otras complicaciones fueron 4 linfoceles precisando tratamiento quirúrgico 3 de ellos y un hematoma perirenal que no precisó cirugía. No hubo complicaciones urológicas. En total se perdieron 6 injertos (15\%) por las complicaciones quirúrgicas. La supervivencia actuarial del injerto fue del $65 \%$ al año, $40 \%$ a los 5 años y $28 \%$ a los 10 .

Conclusiones: La supervivencia de los $3^{\circ}$ y $4^{\circ}$ trasplantes renales es menor que la de los $1^{\circ}$ y $2^{\circ}$. La cirugía mediante abordaje iterativo es dificultosa y se asocia a un mayor índice de complicaciones vasculares. Se precisan estudios multicéntricos con un mayor número de pacientes para poder concluir qué pacientes se beneficiarían de los múltiples retrasplantes.

Palabras clave: Trasplante renal. Retrasplante. Complicaciones quirúrgicas.

\section{ABSTRACT}

\section{SURGICAL ASPECTS IN THE THIRD AND FOURTH KIDNEY RETRANSPLANT}

Introduction and objectives: A quarter of patients waiting for kidney transplantation are patients with previous graft failure. Outcome of first and second renal transplant make these the gold standard for end renal stage disease, but this is not so clear in the case of third and further renal transplant, especially at the time of organ shortage. We revise our experience in patients with three or more kidney transplants focusing on surgical aspects and graft outcome.

Material and method: 1364 renal transplants have been carried out in our centre since 1975 until December 2003. We have retrospectively revised the 34 patients with three renal transplants and the 5 with four. We analyse the surgical technique, surgical complications and graft outcome.

Results: Mean age was 42 years (21-65). Average mismatches between donor and recipient was 3.2. All kidneys, but one case of living donor, were harvested from cadaver donors, mostly in multiple organ-procurement. Average time from the last renal transplant was 5 years ( 3 days- 17 years) and from the last transplant carried out in the iliac fossa reused until the new transplant was 9 years ( 3 days-17.5 years). All implants were performed through an iterative lumboliliac incision ( 25 on the right side, 11 on the left one and in 3 cases where side was not registered). Mean average duration of the procedure was 166 minutes (100-300). Nephrectomy of previous graft at the moment of the implant was carried out in 13 patients (33\%). Vascular anastomosis was made on the common iliac vessels $(50 \%)$ or on the external ones (50\%) in end to side way. Ureteroneocystostomy was performed in an extravesical way except in 1 patient with cutaneous diversion. Vascular complications were 4 haemorrages ( 1 patient died), 3 venous and 2 arterial thrombosis. We had an abscess secondary to intestinal fistulae. Other surgical complications were 4 lymphoceles, three of them needed surgical treatment, and one perirenal haematoma treated in a conservative way. No urological complications were seen. In total 6 grafts (15\%) were lost due to surgical complications. Graft actuarial survival rate at 1 year was $65 \%, 40 \%$ at 5 and $28 \%$ at 10 years.

Conclusions: Three and four renal transplant survival rates are shorter than first and second ones. Iterative access through lumboiliac incision is associated with a higher vascular complication rate, probably in these patients a transperitoneal access would be better. Multicentric studies with higher numbers of patients are needed to define more clearly which patients would benefit from multiple kidney retransplants.

Keywords: Kidney transplant. Retransplant. Surgical complications. 
$\mathrm{E}$ fracaso de un injerto previo es una de las causas más frecuentes de insuficiencia renal, constituyendo aproximadamente un 25\% de los pacientes en lista de espera para trasplante renal ${ }^{1-2}$. El pronóstico de estos retrasplantes está influido por múltiples factores siendo considerados en general de alto riesgo tanto por las posibles complicaciones inmunológicas como por las dificultades quirúrgicas, especialmente a partir del tercer injerto ${ }^{1-3}$.

\section{OBJ ETIVO}

Analizar los aspectos y complicaciones quirúrgicas en nuestra serie de terceros y cuartos restrasplantes y la evolución de los mismos.

\section{MATERIAL Y METODOS}

Estudio retrospectivo de los 39 pacientes multitrasplantados renales (34 con $3^{\text {er }}$ trasplante y 5 con $4^{\circ}$ ). Se analizan las características de los pacientes, causa de pérdida de los injertos previos, aspectos quirúrgicos, complicaciones de la cirugía y evolución de los injertos. No se valoró la inmunosupresión utilizada ya que el período va desde 1981 a 2003 y a lo largo de él ha ido modificándose acorde a los nuevos fármacos y protocolos utilizados.

Los datos se introdujeron en una base de datos Excel y el estudio estadístico se realizó con el paquete estadístico SPSS. Las comparaciones entre las variables cuantitativas se realizaron con el test de la t de Student y las cualitativas con el test de la CHI cuadrado. La supervivencia se valoró mediante curvas de Kaplan-Meier comparándolas mediante el log-rank test.

\section{RESULTADOS}

Entre 1975 y diciembre de 2003 realizamos en nuestro Hospital 1364 trasplantes de riñón, de los que $34(2,5 \%)$ fueron terceros y $5(0,37 \%)$ cuartos.

20 eran pacientes varones y 14 mujeres, la edad media fue 42 \pm 11 años (rango 21-65).

La supervivencia del primer trasplante osciló entre 0 y 11 años $(X=2,2 \pm 3.4$ años $)$ y la del segundo entre 0 y 17,6 años $(X=2,5 \pm 0.3)$. La causa de pérdida de estos injertos fue vascular en 13 casos (19,1\%), rechazo agudo en $20(29,4 \%)$, crónico en $33(48.6 \%)$ y recidiva de la enfermedad primaria en $2(2,9 \%)$.
Entre el $2^{\circ}$ y 3 er trasplante pasaron $5 \pm 3,5$ años (rango 3 días-17 años) habiendo estado en diálisis desde el fallo del $2^{\circ}$ una media de $2,3 \pm 2$ años (rango 0 días-9 años) y entre el $3^{\circ}$ y $4^{\circ}$ pasaron 6,3 $\pm 2,3$ años (rango 4,3-10) habiendo permanecido en diálisis una media de 2,5 \pm 2.7 años (rango 159 días-7,6 años).

El tiempo desde que se hizo el primer trasplante en la fosa iliaca utilizada para el tercero o cuarto trasplante y la realización del mismo fue de $9 \pm 5$ años (rango 3 días y 17,5 años).

El número de trasfusiones previas era de $14,75 \pm 15,4$; el porcentaje de anticuerpos máximo fue $37,15 \pm 33,5$ y actuales $16,3 \pm 25,9$ y el número de incompatibilidades fue $3,16 \pm 1,15$.

Excepto un caso de donante vivo todos los demás injertos procedieron de donantes cadáveres y en la mayoría de los casos de extracciones multiorgánicas; la edad media de los donantes fue de 40,6 $\pm 15,9$ años (12-70). El tiempo de isquemia fría medio fue de $22,13 \pm 5,12$ horas (40 minutos-33 horas).

Se implantaron 20 injertos derechos $(51,3 \%)$, y 14 izquierdos (35,9\%), en 5 casos no consta el injerto. Se utilizó preferentemente la fosa ilíaca derecha (25 pacientes, $64,10 \%$ ) respecto de la izquierda ( 11 pacientes. $28,2 \%$ ), no constando el lado utilizado en 3 casos.

$\mathrm{El}$ abordaje quirúrgico fue a través de una incisión lumboiliaca extraperitoneal iterativa. En 13 pacientes (33\%) se hizo trasplantectomía simultáneamente al implante del injerto. La anastomosis arterial se hizo en término-lateral a los iliacos externos en 16 pacientes, comunes en 18, aorta en 1 caso y no consta en 4; en 30 pacientes fue una única arteria, doble en 5 y no consta en 4 . La anastomosis venosa se hizo a vena iliaca común en 17 pacientes, a externa en 18 y no consta en 4; en 31 pacientes fue vena única, doble en 4 y no consta en 4. El uréter se reimplantó mediante técnicas extravesicales (16 Woodruf, 17 TaguchiVillavicencio, 1 Barry, no consta en 4) excepto en un paciente en que se hizo cutánea; se utilizó catéter doble $\mathrm{J}$ en 10 pacientes. La duración de la intervención fue de 165,8 $\pm 37,9$ minutos (rango 100-300) sin que hubiera diferencias significativas entre los pacientes con trasplantectomía previa $(166,9 \pm 56,3)$ o coincidente con el trasplante

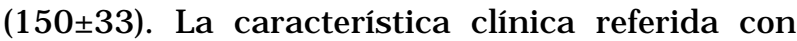


más frecuencia en el protocolo quirúrgico fue la fibrosis. En 23 pacientes hubo diuresis inmediata, no precisando diálisis en los primeros siete días 19 de ellos. Las complicaciones quirúrgicas fueron fundamentalmente vasculares (Tabla I), siendo llamativo el hecho de que en esta serie no se produjeran complicaciones urológicas. Hubo 1 caso de fallecimiento $(2,5 \%)$ por hemorragia masiva en paciente con anastomosis arterial a aorta y ureterostomía cutánea. De los 4 linfoceles tres precisaron tratamiento quirúrgico. $\mathrm{El}$ hematoma perirenal fue tratado de forma conservadora. Una paciente desarrolló una fístula entero-cutánea con posterior absceso y pérdida del injerto por infección debido a la apertura accidental de un asa de delgado durante el trasplante pese a que se suturó en el mismo acto. Los 5 casos de trombosis vascular conllevaron la pérdida del injerto, el cuál fue extirpado entre 0 y 4 días. En total se perdieron 6 injertos por complicaciones quirúrgicas $(15,4 \%)$.

En la actualidad viven con injerto funcionante 15 pacientes, 4 fallecieron por otras causas con injerto funcionante, 7 se perdieron por rechazo agudo y 8 por crónico, 5 por problemas vasculares y 1 por infección. La supervivencia actuarial del injerto al año es del 65\%, a los 5 años del 40\% y a los 10 del $28 \%$.

\section{Tabla I}

Complicaciones quirúrgicas

\begin{tabular}{lcr}
\hline Complicación & N & \% \\
Hemorragia & 4 & 10,25 \\
Trombosis arterial & 2 & 5,12 \\
Trombosis venosa & 3 & 7,69 \\
Hematoma peri renal & 1 & 2,56 \\
Linfocele & 4 & 10,25 \\
Fistula entero-cutánea & 1 & 2,56 \\
Absceso lecho quirúrgico & 1 & 2,56 \\
Exitus & 1 & 2,56
\end{tabular}

\section{DISCUSION}

Entre la tercera y cuarta parte de los pacientes que pierden un injerto renal vuelven a la lista de espera para trasplante y constituyen entre el 15 y $17 \%$ de los trasplantes que se realizan $^{1-5 .}$ El empeoramiento del estado general de los pacientes, la propia decisión del paciente y la falta de remisión desde sus centros son las principales causas de no retorno a la lista de espera ${ }^{4}$.

Se acepta que la supervivencia a largo plazo de los retrasplantes es inferior a la de los primeros $^{6}$ pese a la mejoría sensible lograda con una mejor selección de los pacientes y el uso profiláctico de OKT3 ${ }^{7-13}$. Se han invocado múltiples factores pronósticos en la supervivencia de los retrasplantes, tales como la propia experiencia del centro trasplantador, edad del donante, donante vivo, supervivencia del injerto previo y causa de pérdida del mismo (si fue por causa vascular o inmunológica a partir del 6o mes no influiría), presencia de anticuerpos y número de incompatibilidades y la trasplantectomía del injerto previo $5,8,14-19$, algunos autores abogan por el retrasplante precoz, incluso antes de volver a diálisis como una estrategia para mejorar la evolución del retrasplante ${ }^{20}$.

En el caso de los terceros y cuartos trasplantes se asocia además el hecho de ser pacientes, en general, con mayor deterioro físico y una mayor dificultad técnica derivada de la reutilización de campos quirúrgicos ya operados, a veces en varias ocasiones, lo que condiciona un mayor tiempo de revascularización ${ }^{21}$. La ubicación del retrasplante dependerá de factores como la ubicación previa de los injertos, existencia previa de trasplantectomía (en nuestra serie la realización de la misma durante el trasplante no influyó tan siquiera en la duración de la intervención), otras complicaciones quirúrgicas previas, estado vascular del receptor e inclusive la propia preferencia del cirujano ${ }^{22}$. En nuestra serie se eligió la fosa ilíaca en función de las cirugías previas y estado vascular del paciente por lo que la mayoría de los terceros trasplantes se hicieron en la fosa ilíaca derecha que fue donde estuvo el primer injerto, se utilizó el abordaje clásico liberando el eje vascular y haciendo la anastomosis en la zona que parecía más apta para ello. Se ha descrito el implante en una zona lumbar baja anastomosando los vasos a la porción superior de los ilíacos comunes o a la vena cava, a través de un abordaje lumboilíaco clásico a través de la incisión previa como hemos hecho nosotros o utilizando un abordaje transperitoneal lo que evita la disección en un tejido fibroso per- 
mitiendo además explorar directamente los ejes vasculares de ambos lados y seleccionar el lugar más idóneo para la anastomosis vascular ${ }^{23,24}$, o bien optar por un trasplante ortotópico ${ }^{18}$. Esta mayor dificultad técnica con un mayor tiempo de revascularización suele conllevar en los terceros trasplantes un mayor retraso en la función inicial del injerto que en la serie de Langley alcanzó un $63 \%$ de los terceros trasplantes ${ }^{21,25}$; en nuestra serie los terceros y cuartos trasplantes requirieron un mayor número de sesiones de hemodiálisis post-trasplante $(7,5 \pm 11,2)$ que los primeros $(5,6 \pm 1,5)$ y segundos $(4,25 \pm 3,2)$ sin que estas diferencias fueran significativas. Así mismo la reutilización de fosas ilíacas para un nuevo injerto renal aumenta la incidencia de complicaciones quirúrgicas tanto vasculares como urológicas hasta un $15 \%^{23,26}$. En general se describen complicaciones vasculares entre un 4 y $12 \%$ de los pacientes trasplantados las cuales son responsables de la pérdida del injerto entre el $6,5 \mathrm{y}$ $17 \%{ }^{27-30}$; en nuestra serie de multiretrasplantados renales éstas ascendieron a un 25\% (hemorragias y trombosis vasculares) las cuales provocaron la pérdida del 13\% de los injertos y 1 fallecimiento $(2,5 \%)$, mientras que Badet et al. ${ }^{23}$ en terceros trasplantes sólo refieren la pérdida de 1 injerto de 23 debido al $9 \%$ de complicaciones vasculares; la utilización de heparina a dosis bajas seguida de la administración profiláctica de ácido acetil salicílico o cumarínicos en pacientes que perdieron el injerto previo por trombosis parece igualar el riesgo de la misma a la de los primeros trasplantes sin incrementar el de hemorragia $^{31,32}$. También se observa una mayor incidencia de linfoceles en los casos de terceros y cuartos trasplantes, $11,7 \%$, frente a la de los primeros y segundos, 4,3-10\%30,32-34.

La supervivencia del tercer trasplante al año oscila entre el 58 y $88 \%, 66 \%$ a los 5 y $38 \%$ a los 10 años $^{23,35}$, en nuestra serie fue del $65,40 \mathrm{y}$ $28 \%$ respectivamente. Matas et al. ${ }^{10}$ encuentran una supervivencia similar en los casos de 3er trasplante a la de los primeros y segundos cuando se hace una adecuada selección de los pacientes, y pese al mayor riesgo de complicaciones quirúrgicas, sus pacientes, especialmente los más jóvenes, consideraron acertada la decisión del tercer trasplante.

\section{CONCLUSIONES}

La supervivencia de los terceros y cuartos trasplantes renales es inferior a la de los primeros y segundos.

El acceso a través de una incisión lumboilíaca iterativa es dificultoso y conlleva un aumento de las complicaciones vasculares; probablemente en estos pacientes sería mejor un acceso transperitoneal.

Estudios multicéntricos con un mayor número de pacientes permitirían definir mejor qué pacientes se beneficiarían de los retrasplantes múltiples.

\section{REFERENCIAS}

1. Cotorruelo JG, Arias M. Lista de espera para trasplante renal. Selección de la pareja donante-receptor, en LH Hernández Avendaño Nefrología clínica (1 $1^{a}$ edición), Edit. Panamericana, Madrid, 1997;686-692.

2. Saladié JM, Lauzuzica R, Escalera C. Retrasplante, en M González-Martín y JM García Butirón Trasplante renal, Edit Aula Médica, Madrid, 2000;429-435.

3. Kahan BD, Mickey R, Flechner SM, Lorber MI, Wideman CA, Kerman RH, Terasaki P, Van Buren CT. Multivariate analysis of risk factors impacting on immediate and eventual cadaver allograft survival in cyclosporine-treated recipients Transplantation 1987;43:65-70

4. Gallichio MH, Hudson S, Young CJ, Diethelm AG, Deierhoi $\mathrm{MH}$. Renal retransplantation at the University of Alabama at Birmingham: Incidence and outcome. Clin Transpl 1998;12:169-175.

5. Candinas D, Weber M, Inderbitzin D, Schlumpf R, Largiader F. Kidney retransplantation in relation to organ shortage. Langenbecks Arch Chir Suppl Kongressbd 1998;115:1571-1573.

6. Moss A, Najarian JS, Sutherland DE, Payne WD, Gruessner RW, Humar A, Kandaswamy R, Gillingham KJ, Dunn DL, Matas AJ. 5000 kidney transplants, a single center experience. Clin Transp 2000;14:159-171.

7. Cho YW, Cecka JM. Cadaver-donor renal retransplants. Clin Transpl 1993;7:469-484.

8. Almond PS, Matas AJ, Gillingham K, Troppmann C, Payne W, Dunn D, Sutherland D, Najarian JS. Risk factors for second renal allograft immunosupressed with cyclosporine. Transplantation 1991;52:253-258.

9. UK Nacional Transplant Database retransplantation in the UK and Republic of Ireland, 1987-1996. UKT SSA Nacional Transplant Database. Clin Transp 1997;11:81-86.

10. Matas AJ, Gillingham KJ, Payne WD, Dunn DL, Gruessner RW, Sutherland DE, Schmidt W, Najarian JS. A third kidney transplant: cost-effective treatment for end-stage renal disease?. Clin Transp 1996;10:516-520.

11. Morris PJ. Results of kidney transplantation en PJ Morris Kidney transplantation, principles and practice, 5th edition, edit by WB Saunders Company, Philadelphia (USA) 2001;693-715.

12. Mitsuishi Y, Cecka JM. Recent improvements in cadaverdonor kidney retransplantation. Clin Transpl 1991;5:281291. 
13. Mouquet C, Benalia H, Chartier-Kastler E, Sylla C, Coriat P, Bitker MO, Richard F. Renal retransplantation in adults. Comparative prognostic study. Progr Urol 1999;9: 239-243.

14. Gjertson DW. A multi-factor analysis of kidney regrafts outcomes. Clin Transpl 2002;16:335-349.

15. Cecka JM. The UNOS Scientific renal transplant registry. Clin Transpl 1998;12:1-6.

16. Abouljoud MS, Deierhoi MH, Hudson SL, Diethelm AG. Risk factors affecting second renal transplant outcome, with special reference to primary allograft nephrectomy. Transplantation 1995;60:138-144.

17. Cabacungan C. Regrafts. Clin Tranpl 1992;6:347-356.

18. Pérez MJ, Rabadán L, Hernández C, et al. Retrasplante. Clin Urol de la Complutense 1999;7:379-384.

19. Douzdjian V, Rice JC, Carson RW Gugliuzza KK, Fish JC. Renal retransplants: effect of primary allograft nephrectomy on early function, acute rejection and outcome. Clin Transpl 1996;10:203-208.

20. Arias M, Escallada R, de Francisco AL, Rodrigo E, Fernandez-Fresnedo G, Setien MA, Pinera C, Ruiz JC, Herraez I, Cotorruelo J. Return to dialysis after renal transplantation: Which would be the best way?. Kidney International 2002;61:85.

21. Montañés P, Cruz N, Torubia F. Infecciones en el trasplante renal (infección del lecho quirúrgico y vías urinarias), en M González-Martín y JM García Buitrón Trasplante renal $1^{\underline{a}}$ edición, edit Aula Médica, Madrid (España), 2000;323-343.

22. Saladié JM, Lauzuzica R, Escalera C. Retrasplante en M González-Martín y JM García Buitrón Trasplante renal $1^{\text {a }}$ edición, edit Aula Médica, Madrid (España), 2000;429-435.

23. Badet L, Lefrancois N, Farsi-Fehri H, et al. Third renal transplantation: is it really the best option?. Eur Urol Suppl 2004;3,4(abstract 5).

24. Malone M, Bihrle W, Libertino JA: Surgical procedures for renal transplantation, bench surgery and autotransplantation in John A Libertino Reconstructive Urologic Surgery 3rd edition, edit Mosby, Saint Louis (USA), 1998;93-100.

25. Langley J, Vergne-Marini P, Dickerman RM, Brinker KR, Land G.: Single center experience of the effect of delayed function on outcome in retransplant recipients of cadaver renal allograft. Tranplant Proc 1991;23:1757-1759.
26. Pertusa C, Arruza A, Zabala JA. Coste y calidad de vida en el trasplante renal en M González-Martín y JM García Buitrón Trasplante renal $1^{\underline{a}}$ edición, edit Aula Médica, Madrid (España), 2000;455-466.

27. Galmes I, Burgos FJ, Borrego J, et al. Complicaciones vasculares en el trasplante renal. Actas Urol Esp 1995;19:814.

28. Montañés P, Sánchez E, Torrubia FJ: Complicaciones quirúrgicas del trasplante renal. Tema monográfico del LVII Congreso Nacional de Urología, ENE Ediciones SA, Madrid (España), 1992;115-132.

29. De la Peña E, Blázquez J, Resel L. Complicaciones vasculares del trasplante renal. Clin Urol de la Complutense 1999;7:555-579.

30. Gil P, Liedana JM, Roncales A et al.: Análisis de nuestra serie de trasplantes renales: complicaciones urológicas y supervivencia. Actas Urol Esp 1998;22:326-335.

31. Humar A, Key N, Ramcharan T, et al. Kidney retransplants after initial graft lost to vascular thrombosis. Clin Transpl 2001;15:6-10.

32. Robertson AJ, Norgund V, Gray DW, et al. Low dose aspirin as prophylaxis against renal vein thrombosis in renal transplant recipients. Nephrol Dial Transplant 2000;15:1865-1868.

33. De la Peña E, Blázquez J, Gómez A: El linfocele en el trasplante renal. Clin Urol de la Complutense 1999;7:581595.

34. Gray DWR:Vascular and lymphatic complications after renal transplantation en PJ Morris Kidney transplantation, principles and practice, 5th edition, edit by WB Saunders Company, Philadelphia (USA) 2001;419-434.

35. Ogura K, Cecka JM: Cadaver retransplants. Clin Transpl 1990;4:471-483.

Dr. J.L. Gutiérrez Baños

Francisco de Cáceres $1 \mathrm{~L}, 4^{\circ} \mathrm{C}$

39012 Santander

E-mail: jlgb@ono.com

(Trabajo recibido el 26 octubre de 2004) 